\title{
Predicción de temperatura corporal usando termómetro infrarrojo en un hato de alpacas (Arequipa, Perú)
}

\author{
Prediction of body temperature using infrared thermometer in an alpaca herd \\ (Arequipa, Peru)
}

\author{
Gabriela Eduardo V. ${ }^{1,2}$, Jorge Vilela V. ${ }^{1}$
}

\section{Resumen}

\begin{abstract}
Se evaluó la temperatura infrarroja corporal de 50 alpacas en un hato de 155 alpacas Huacaya macho en el Centro de Desarrollo Alpaquero (CEDAT - Toccra) en Arequipa, Perú. Se utilizó un termómetro infrarrojo sobre las fosas nasales, zona interna de la oreja, zona media de la oreja y vientre y un termómetro rectal para medir la temperatura corporal, Además, se registró la temperatura ambiental. Se buscó determinar mediante regresión el mejor modelo predictivo de temperatura corporal en alpacas. Los modelos predictivos mostraron que las variables de temperatura de la zona de la oreja interna y temperatura ambiental dan una mejor predicción de la temperatura corporal en alpacas. Se concluye que es posible predecir la temperatura corporal usando termografía infrarroja.
\end{abstract}

Palabras clave: alpaca, temperatura corporal, termómetro infrarrojo, predicción

\section{Abstract}

The infrared body temperature of 50 alpacas was evaluated in a herd of 155 male Huacaya alpacas at the Alpaca Development Center (CEDAT - Toccra) in Arequipa, Peru. An infrared thermometer was used on the nostrils, inner ear zone, middle ear zone and abdomen and a rectal thermometer to measure body temperature. Besides, ambient temperature was also recorded. The best predictive model of body temperature in alpacas

\footnotetext{
${ }^{1}$ Universidad Científica del Sur, Lima, Perú

${ }^{2}$ E-mail: natalia.eduardo.villagra@gmail.com
}

Recibido: 18 de octubre de 2020

Aceptado para publicación: 20 de septiembre de 2021

Publicado: 27 de octubre de 2021

CLos autores. Este artículo es publicado por la Rev Inv Vet Perú de la Facultad de Medicina Veterinaria, Universidad Nacional Mayor de San Marcos. Este es un artículo de acceso abierto, distribuido bajo los términos de la licencia Creative Commons Atribución 4.0 Internacional (CC BY 4.0) [https:// creativecommons.org/licenses/by/4.0/deed.es] que permite el uso, distribución y reproducción en cualquier medio, siempre que la obra original sea debidamente citada de su fuente original 
was sought to be determined by regression analysis. The predictive models showed that the temperature variables of the inner ear zone and environmental temperature give a better prediction of body temperature in alpacas. It is concluded that it is possible to predict body temperature using infrared thermography.

Key words: alpaca, body temperature, infrared thermometer, prediction

\section{INTRODUCCIÓN}

En las zonas más altas y aisladas del Perú se encuentran las comunidades campesinas y pequeños productores dedicados a la crianza de alpacas. Estas comunidades, según Siguayro (2009), son parte importante de la cultura y economía del país. En el Perú, según el último censo nacional agropecuario, se cuenta con una población aproximada de 3.7 millones de alpacas, estando la mayor población en las regiones de Puno, Cusco y Arequipa (CENAGRO, 2012).

El manejo sanitario de los rebaños es de extrema importancia en la crianza de animales, de allí que los criadores de alpacas deben reconocer enfermedades y problemas sanitarios en el momento adecuado (Sellier et al., 2017). Una de las maneras para identificar a tiempo y evaluar de manera recurrente el estado sanitario de las alpacas es mediante la medición de las variables fisiológicas, siendo el parámetro más usado en medicina veterinaria la temperatura corporal (Heath et al., 2001).

El aumento de la temperatura corporal puede deberse a distintos motivos; entre estos a cambios en las funciones metabólicas como la respiración, alimentación y desplazamiento de los animales (Heath et al., 2001). Las alpacas recurren a distintos mecanismos para dispersar el calor cuando se presenta un incremento de la temperatura corporal; sin embargo, estos pueden fracasar cuando no hay movimiento del aire y la temperatura corporal llega a ser similar a la temperatura ambiental. Es así, que cambios en los valores de temperatura corporal, pueden ser un indicador de alteración en el estado de la salud del paciente. Poikalainen et al. (2012) indican que al ser un indicador importante en el diagnóstico es necesario que su medición sea precisa y clara.

La temperatura rectal se puede usar como un indicador de estrés térmico o incluso como indicador de enfermedad (Mostaco et al., 2015). En la práctica veterinaria, la medición de temperatura rectal es la más común, siendo considerada como el «patrón de oro» por la conexión que existe con la temperatura corporal central (Gomart et al., 2014). Por otro lado, introducir el termómetro rectal puede provocar estrés en el animal, el cual se traduce en un comportamiento de defensa. Hanneman et al. (2004) mencionan que la introducción del termómetro rectal puede provocar estrés en el animal, por lo que es necesario utilizar técnicas no invasivas para la medición y de fácil aplicación para mantener el bienestar animal durante la obtención de temperatura corporal. Ante esto y dentro de los métodos alternativos se dispone de la termometría infrarroja que mide la temperatura sin contacto con el cuerpo, ofreciendo varios beneficios a comparación de la temperatura rectal (Soerensen y Pedersen, 2015). Según Chung et al. (2010) tiene como ventajas su precisión $\left( \pm 0.1{ }^{\circ} \mathrm{C}\right)$, tiempo de medición menor a 0.5 segundos y ausencia de necesidad de contacto, siendo así que se requiere menor sujeción y manejo del animal, reduciendo así el riesgo de propagación de infecciones y estrés en el animal. 
En esta investigación se busca predecir la temperatura corporal en alpacas usando termómetro infrarrojo, y así emplear menor sujeción y manejo disminuyendo la molestia y estrés en el animal, obteniendo valores precisos y rápidos en menor tiempo.

\section{Materiales y Métodos}

El estudio se realizó en junio de 2019 en Toccra, distrito de Yanque, provincia de Caylloma, Región Arequipa. El CEDATToccra es un centro de cría y banco de reproductores de alpacas y llamas, ubicado a una altitud de $4400 \mathrm{~m}$. Las condiciones de crianza son de puna seca con precipitación pluvial anual de $350 \mathrm{~mm}$, temperatura ambiental entre -20.0 y $18.0^{\circ} \mathrm{C}$ y alimentación al pastoreo natural de tipo pajonal de bajo valor nutricional.
El estudio se realizó en junio de 2019 en Toccra, distrito de Yanque, provincia de Caylloma, Región Arequipa, ubicado a 4400 msnm. La temperatura ambiental durante el estudio estuvo entre -20.0 y $18.0{ }^{\circ} \mathrm{C}$ y las pasturas eran de pobre calidad y cantidad.

El tipo de muestreo fue irrestricto aleatorio. Se utilizó un tamaño poblacional de 155 animales, error $=0.1^{\circ} \mathrm{C}$, varianza estimada $=$ $0.19{ }^{\circ} \mathrm{C}$ y nivel de confianza de $95 \%$, obteniéndose un tamaño muestral mínimo de 49.8 animales. De esta forma, se trabajó con 50 alpacas macho con edades entre 1 y 13 años, que fueron seleccionadas en forma aleatoria de la población Huacaya del centro de recría. La temperatura corporal fue tomada por un operario mientras otra persona sujetaba al animal. La temperatura corporal se midió con un termómetro rectal clínico de mercurio Level y un termómetro infrarrojo Fluke 62 max (Fluke Corporation), de mane-
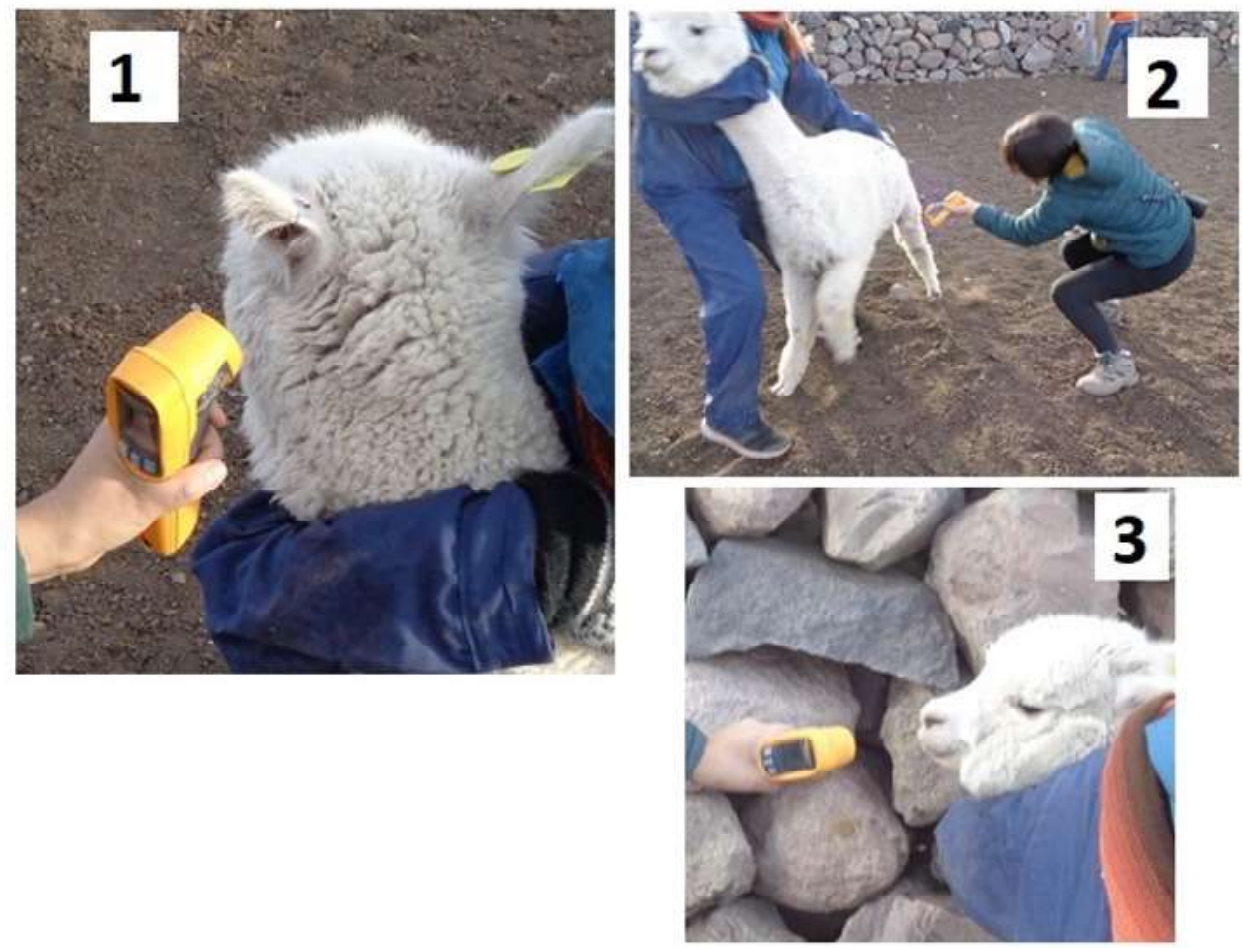

Figura 1. Medicion de temperatura corporal en alpacas con termometro intrarrojo. 1: Medición en la zona de la oreja. 2: Medición en la zona del vientre. 3: Medición en la zona de fosas nasales 
ra simultánea entre las 06:00 y las 18:00 h dentro del corral y al aire libre en condiciones de temperatura ambiental no controlada. La temperatura mínima al momento de la evaluación fue de $-7.5^{\circ} \mathrm{C}$ y la máxima de $19.5^{\circ} \mathrm{C}$. Además, en cada ocasión se registró la temperatura ambiental con un termómetro Hakusa Hks (República Checa) (-50 a $70{ }^{\circ} \mathrm{C}, \pm 1{ }^{\circ} \mathrm{C}$ ).

El termómetro rectal tiene un rango de medición entre 35.2 y $42.2^{\circ} \mathrm{C}$ y fue insertado aproximadamente de 6 a $7 \mathrm{~cm}$ en el recto durante un minuto. El termómetro infrarrojo tiene una precisión de $\pm 0.1{ }^{\circ} \mathrm{C}$, rango de acción de $23-510^{\circ} \mathrm{C}$, emisividad de 0.1 a 1.0 , velocidad de medición menor a $0.5 \mathrm{~s}$. La medición de la temperatura se hizo a una distancia menor de $30 \mathrm{~cm}$ en las zonas de las fosas nasales, zona interna de la oreja, zona media de la oreja y el vientre, que representa un área igual o menor a $5.88 \mathrm{~cm}^{2}$, como se indica en la Figura 1. Las variables utilizadas fueron temperatura rectal (TR), temperatura infrarroja de las zonas de fosas nasales (FN), zona interna de la oreja (OI), zona media de la oreja $(\mathrm{OM})$, vientre $(\mathrm{V})$ y temperatura ambiental (TA

Todos los procedimientos y cuidados se llevaron a cabo siguiendo la Ley de Protección y Bienestar Animal N. ${ }^{\circ} 30407$ de la República del Perú y de acuerdo con las recomendaciones del Código de Salud de los Animales Terrestres de la Organización Mundial de Sanidad Animal (OIE, s.f.). El proyecto de investigación fue aprobado por el Comité Institucional de Ética en investigación con animales y biodiversidad de la Universidad Científica del Sur con el código de registro 047-2019-PRE16.

Para el análisis de datos se utilizó el paquete estadístico $\mathrm{SAS} \AA$, con el comando de función PROC REG y PROC CORR, para el análisis de regresión múltiple y la correla- ción de Pearson entre todas las variables, respectivamente. La variable dependiente TR fue sometida a la prueba de Anderson Darling (1952) para comprobar los supuestos de normalidad. El modelo de regresión propuesto fue: $Y=\mu+\beta 0+\beta 1 \times 1+\beta 2 \times 2+\beta 3 \times 3+\beta 4 \times 4$ $+\beta 5 \times 5$, donde $\mathrm{Y}=$ Variable temperatura rectal $\left({ }^{\circ} \mathrm{C}\right), \mu=$ Promedio, $\beta 0=$ Intercepto, $\beta 1=$ Coeficiente de regresión del efecto de la variable temperatura infrarroja (TI) de FN, $\beta 2$ $=$ Coeficiente de regresión del efecto de la variable TI de OI, $\beta 3=$ Coeficiente de regresión del efecto de la variable TI de OM, $\beta 4=$ Coeficiente de regresión del efecto de la variable $\mathrm{TI}$ de $\mathrm{V}, \beta 5=$ Coeficiente de regresión del efecto de la variable TA, X1 = Efecto de la variable TI de FN, X2 = Efecto de la variable TI de OI, X3 = Efecto de la variable TI de OM, X4 = Efecto de la variable TI de V, $\mathrm{X} 5=$ Efecto de la variable TA.

Este modelo fue sometido a análisis de regresión múltiple para determinar el mejor modelo predictivo, donde se usó como criterio de inclusión en el modelo las variables con nivel de significancia menor o igual a $0.5 \mathrm{y}$ por el método «Forward» se incluyeron las variables de uno en uno. Solo se analizaron los modelos predictivos que cumplían con el criterio de inclusión. Para escoger el mejor modelo predictivo se consideró el coeficiente de determinación $\left(\mathrm{R}^{2}\right)$ que indica la proporción de variación de los resultados que puede ser explicada por el modelo, el Coeficiente de Mallows (Cp) que determina el sesgo del modelo, el criterio de Akaike (AIC) que estima el error de predicción de la muestra, el criterio de información Bayesiana (BIC) y el criterio de información Bayesiana de Schwarz (SBC) que tiene función similar a AIC en estadística bayesiana. La comparación entre las temperaturas rectal y temperatura predicha por el modelo elegido fue evaluada mediante el coeficiente de correlación de concordancia (CCC) con intervalo de confianza al 95\%. 
Cuadro 1. Medición de temperatura ambiental y temperatura corporal de alpacas con termómetro rectal y termómetro infrarrojo ( $\mathrm{n}=50$ alpacas)

\begin{tabular}{lcc}
\hline $\begin{array}{l}\text { Temperatura } \\
\left({ }^{\circ} \mathrm{C}\right)\end{array}$ & Media \pm D.E. & $\begin{array}{l}\text { CV } \\
(\%)\end{array}$ \\
\hline T. rectal & $37.92 \pm 0.52$ & 1.37 \\
T. ambiental & $11.32 \pm 7.15$ & 63.16 \\
T. fosas nasales & $27.93 \pm 3.58$ & 12.82 \\
T. oreja interna & $22.77 \pm 6.66$ & 29.25 \\
T. oreja media & $21.61 \pm 8.35$ & 38.64 \\
T. vientre & $27.47 \pm 4.86$ & 17.69 \\
\hline
\end{tabular}

\section{Resultados}

Los resultados de las temperaturas tomadas con el termómetro rectal, termómetro ambiental y termómetro infrarrojo se muestran en el Cuadro 1. La significancia de TR para la prueba de Anderson Darling fue de $\mathrm{p}=0.09$. Las correlaciones de la temperatura rectal con la temperatura ambiental e infrarrojas se muestran en el Cuadro 2.

De acuerdo con el análisis de correlación, la temperatura ambiental tuvo la más alta correlación positiva con la temperatura rectal. La temperatura de las fosas nasales tuvo una correlación media con la medida de temperatura rectal, mientras que, con las variables de temperatura de zona interna de la oreja, zona media de la oreja y vientre se observaron valores de correlación negativas.

Los resultados del análisis de regresión múltiple se encuentran en la Figura 2. Los modelos que presentaron valores más bajos de Criterio de Akaike (AIC), Información Bayesiana (BIC), Coeficiente de Mallows (Cp) e Información Bayesiana de Schwarz (SBC) fueron los modelos 2 y 3 . No fueron incluidos en el análisis los modelos que incluyeron las variables de FN y OM por no presentar una significancia menor a 0.5 . De otro lado, el coeficiente de determinación ajustado ( $\mathrm{R}^{2}$ ajustado) más alto fue considerado como la mejor opción, por lo que se considera al modelo 2 (OI, TA) como el mejor de los tres modelos propuestos (Cuadro 3). De esta manera, el mejor modelo para la predicción de temperatura rectal incluye a las variables de temperatura ambiental y de oreja interna. El coeficiente de correlación de concordancia entre las temperaturas rectales y temperaturas predichas por modelo, son presentadas en el Cuadro 3.

\section{Discusión}

La termometría rectal es el método estándar de medición de la temperatura en la práctica clínica, siendo en algunos casos asociada a un estrés significativo para el paciente. Chen y White (2006) y Chung et al. (2010) han realizado estudios sobre la predicción de la temperatura corporal mediante la toma de temperatura infrarroja en especies como cerdos, conejos, vacas y alpacas.

El mejor modelo se encuentra entre el 2 (OI y TA) y el 3 (OI, V, TA), según el coeficiente de determinación ajustado $\left(\mathrm{R}^{2}\right)$, ya que 
Cuadro 2. Coeficientes de correlación entre los valores de temperatura ambiental, temperatura infrarroja y temperatura rectal en alpacas. ( $\mathrm{n}=50$ alpacas)

\begin{tabular}{lcccccc}
\hline & \multicolumn{6}{c}{ Temperatura $\left({ }^{\circ} \mathrm{C}\right)$} \\
\cline { 2 - 7 } & Ambiental & Rectal & $\begin{array}{c}\text { Fosa } \\
\text { nasal }\end{array}$ & Oreja interna & $\begin{array}{c}\text { Oreja } \\
\text { media }\end{array}$ & Vientre \\
\hline Ambiental & 1 & 0.108 & $0.286^{1}$ & $0.585^{2}$ & $0.576^{2}$ & 0.012 \\
Rectal & 0.108 & 1 & 0.034 & -0.207 & -0.053 & -0.104 \\
Fosa nasal & $0.286^{1}$ & 0.034 & 1 & $0.373^{2}$ & $0.426^{2}$ & -0.169 \\
Oreja interna & $0.585^{2}$ & -0.207 & $0.373^{2}$ & 1 & $0.656^{2}$ & 0.033 \\
Oreja media & $0.576^{2}$ & -0.053 & $0.426^{2}$ & $0.656^{2}$ & 1 & $0.290^{1}$ \\
Vientre & 0.012 & -0.104 & -0.169 & 0.033 & $0.290^{*}$ & 1 \\
\hline
\end{tabular}

${ }^{1}$ La correlación es significativa en el nivel 0.05 (bilateral)

${ }^{2}$ La correlación es significativa en el nivel 0.01 (bilateral)

Cuadro 3. Resultados de coeficiente de correlación de concordancia entre la temperatura predicha y la temperatura rectal de los tres modelos propuestos

\begin{tabular}{lcccccc}
\hline Modelo & $\begin{array}{c}\mathrm{R}^{2} \\
\text { ajustado }\end{array}$ & $\begin{array}{c}\text { Error } \\
\text { estándar } \\
\text { de la } \\
\text { estimación }\end{array}$ & $\begin{array}{c}\text { Significancia } \\
\text { del modelo }\end{array}$ & $\begin{array}{c}\text { Temp. } \\
\text { predicha } \\
\text { promedio } \\
\left({ }^{\circ} \mathrm{C}\right)\end{array}$ & $\begin{array}{c}\text { CCC con } \\
\text { temp. } \\
\text { rectal }\end{array}$ & $\begin{array}{c}\text { I.C. al 95\% } \\
\text { de CCC }\end{array}$ \\
\hline 1 & 0.023 & 0.508 & 0.148 & 37.918 & 0.08 & $-0.03,0.19$ \\
2 & 0.086 & 0.491 & 0.045 & 37.958 & 0.18 & $0.02,0.33$ \\
3 & 0.076 & 0.194 & 0.086 & 37.918 & 0.23 & $0.07,0.39$ \\
\hline
\end{tabular}

Modelo 1: (constante), oreja interna

Modelo 2: (constante), oreja interna, ambiental

Modelo 3: (constante), oreja interna, ambiental, vientre

C.C.C.: Coeficiente de correlación de concordancia

I.C.: Intervalo de confianza

presenta los valores de coeficiente de determinación más altos. Asimismo, teniendo en cuenta los valores del criterio de Akaike, información Bayesiana e información Bayesiana de Schwarz, el mejor modelo sería el 2. Aunque el modelo 3 representa una opción posible, no se le considera debido a que la medición de temperatura del vientre implica mayor sujeción del animal, lo cual hace difícil la medición de la temperatura infrarroja en esa zona. Es así, que el mejor modelo que se encontró para la temperatura rectal usa predictores de la temperatura de zona interna de la oreja y la temperatura ambiental. La temperatura ambiental tiene una distribución errática y sobredispersa y tuvo 
$\mathrm{R}$ - cuadrado
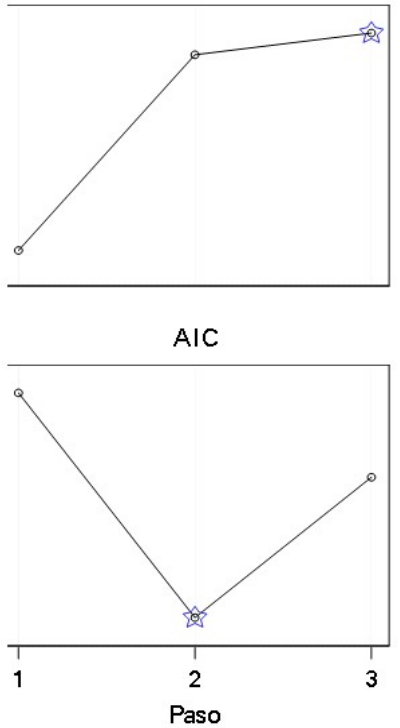

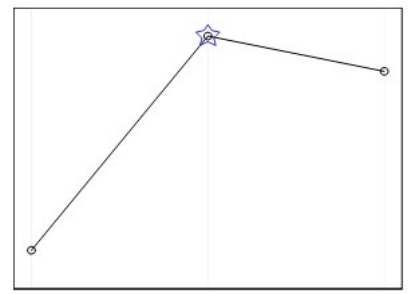

$\mathrm{BIC}$

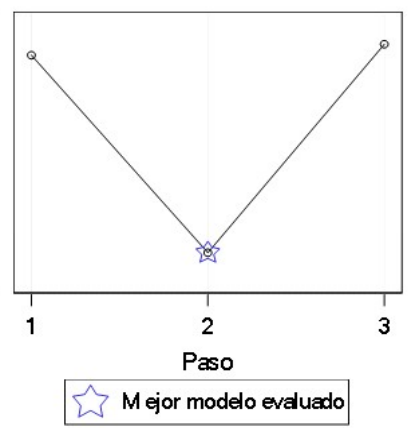

$\mathrm{C}(\mathrm{p})$

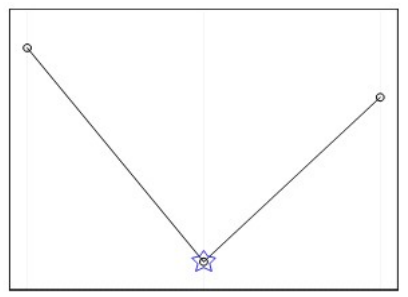

$\mathrm{SBC}$

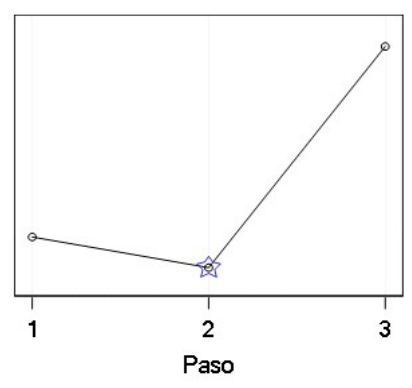

Figura 2. Criterio de ajuste para elegir el mejor modelo para temperatura rectal $C(p)$ : Coeficiente de Mallows, AIC: Criterio de Información de Akaike, BIC: Criterio de Información Bayesiana, SBC: Criterio de Información Bayesiana de Schwarz

una amplia variabilidad durante la toma de temperatura corporal de los animales.

Mostaco et al. (2015) y Vilela (2018) coinciden al indicar que el mejor ajuste fue la temperatura de la oreja a través del termómetro infrarrojo, a diferencia de otras áreas del cuerpo más expuestas. En este sentido, Gomart et al. (2014) mencionan que la termometría infrarroja en el área auricular es una alternativa confiable y menos estresante para estimar la temperatura rectal. Un factor positivo es la practicidad por la ubicación anatómica de la oreja (Kunkle et al., 2004).

Según Kammersgaard et al. (2013), la oreja al ser una extremidad vascularizada funciona como órgano termorregulador, siendo así una buena fuente de información con respecto al estado térmico. Cuppit y Badsha (2002) mencionan que la temperatura en perros tomada en la oreja tiene una estimación altamente precisa de la temperatura central, aunque inferior a la rectal. De otra parte, Tabuaciri et al. (2012) concluyeron que la correlación entre la temperatura de superficie y la temperatura rectal era más alta en la oreja, mientras que Vieira et al. (2016) demostraron que la termografía infrarroja de la frente de las vacas tenía una mayor asociación con la temperatura rectal; sin embargo, en dicho estudio se cepilló el pelo de las vacas para evitar que la suciedad interfiera en la toma de temperatura y el alimento fue restringido por 18 horas para minimizar el efecto del aumento de la temperatura como resultado de la digestión de los alimentos. 
Los resultados del coeficiente de correlación de Pearson (Cuadro 2), indican una correlación muy baja y negativa entre las variables temperatura rectal y temperatura de la zona interna de la oreja, así como una correlación baja entre las variables temperatura rectal y temperatura ambiental. El coeficiente de correlación de concordancia que se realizó entre la temperatura rectal y la temperatura predicha fue de 0.18 , siendo un valor de correlación bajo. Esto pudo deberse a que el ambiente en el que se toma la muestra no es un ambiente controlado, generando que la temperatura ambiental tenga una variabilidad elevada.

De acuerdo con Soerensen y Pedersen (2015), las áreas con correlaciones más altas con la temperatura corporal fueron los ojos y las orejas, dependiendo en el caso de las orejas la ubicación de la medida. Así, la correlación con la zona de la oreja interna fue mayor $(\mathrm{r}=0.85)$ en comparación de la zona media $(r=0.27)$. La variable temperatura ambiental con temperatura de vientre presentan una correlación elevada indicando que esta variable estaría influenciada por el medio ambiente. Por otro lado, la precisión de la medición con el termómetro rectal puede estar influenciada por la experiencia y exactitud del encargado y esto puede generar variaciones entre mediciones, por lo que en estudios donde no se utilizó la medición de la temperatura rectal (se usó la temperatura de la sangre saliente de una herida) los coeficientes de correlación y regresión fueron más elevados (Chen y White (2006).

Loughmiller et al. (2001) indicaron que la condición externa que con mayor frecuencia afecta la temperatura corporal es la temperatura ambiental, debido a que cambios de esta afecta en la vasodilatación del tejido subcutáneo. De otro lado, Knizkova y Kunc (2007) refieren que la temperatura infrarroja tiene diversas limitaciones, tales como el pelaje del animal (la suciedad y la humedad), el tiempo de alimentación, la rumia y la lactación. Sumado a esto, es importante considerar que dentro de las condiciones climáticas en las que se encontraron las alpacas del estudio, es común encontrar un clima frío y con corrientes de aire, generando pérdida de temperatura corporal que pueden alterar las mediciones con termómetro infrarrojo (Kammersgaard et al., 2013).

\section{Conclusión}

No obstante que los resultados de correlación entre temperatura rectal y temperatura de oreja interna de las alpacas fueron negativos, se obtuvo una correlación de concordancia positiva entre la temperatura predicha y la temperatura rectal; de allí que se podría predecir la temperatura corporal de alpacas usando la temperatura infrarroja de la zona interna de la oreja y la temperatura ambiental.

\section{Literatura Citada}

1. Anderson TW, Darling DA. 1952. Asymptotic theory of certain «goodnessof-fit» criteria based on stochastic processes. Ann Mathematical Stat 23: 193-212. doi: 10.1214/aoms/1177729437

2. Chen P, White C. 2006. Comparison of rectal, microchip transponder, and infrared thermometry techniques for obtaining body temperature in the laboratory rabbit (Oryctolagus cuniculus). J Am Assoc Lab Anim 45: 57-63.

3. Chung T, Jung W, Nam E, Kim J, Park S, Hwang C. 2010. Comparison of rectal and infrared thermometry for obtaining body temperature of gnotobiotic pigs in conventional portable germ free facility. Asian Austral J Anim 23: 13641368. doi: 10.5713 /ajas.2010.90507

4. Cuppit J, Badsha Z. 2002. Temperature measurements - which method is best? Anaesthesia 57: 619. doi: 10.1046/ j.1365-2044.2002.265818.x.

5. Gomart S, Allerton F, Gommeren $\mathrm{K}$. 2014. Accuracy of different temperature reading techniques and associated stress 
response in hospitalized dogs. J Vet Emerg Crit Car 24: 279-285. doi: 10.1111/ vec. 12155

6. Hanneman S, Jesurum J, Bickel D. 2004. Comparison of methods of temperature measurement in swine. Lab Anim 38: 297-306. doi: 10.1258/002367704323133682

7. Heath A, Navarre C, Simpkins A, Purohit R, Pugh D. 2001. A comparison of surface and rectal temperatures between sheared and nonsheared alpacas (Lama pacos). Small Ruminant Res 39: 19-23. doi: 10.1016/ s0921-4488(00)00173-5

8. [INEI] Instituto Nacional de Estadística e Informática. 2012. IV Censo Nacional Agropecuario. [Internet]. Disponible en: http://censos.inei.gob.pe/ Cenagro/redatam/

9. Kammersgaard T, Malmkvist J, Pedersen L. 2013. Infrared thermography - a non-invasive tool to evaluate thermal status of neonatal pigs based on surface temperature. Animal 7: 2026234. doi: $10.1017 / \mathrm{S} 1751731113001778$

10. Knizkova I, Kunc P. 2007. Applications of infrared thermography in animal production. J Faculty Agric OMU 22: 329-336.

11. Kunkle G, Nicklin C, Sullivan-Tamboe D. 2004. Comparison of body temperature in cats using a veterinary infrared thermometer and a digital rectal thermometer. J Am Anim Hosp Assoc 40: $42-$ 46. doi: 10.5326/0400042

12. Loughmiller J, Spire M, Dritz S, Fenwick B, Hosni H, Hogge S. 2001. Relationship between mean body surface temperature measured by use of infrared thermography and ambient temperature in clinically normal pigs and pigs inoculated with Actinobacillus pleuropneumoniae. Am J Vet Res 62: 676-681. doi: 10.2460/ajvr.2001.62.676

13. Mostaco G, Miranda K, Condotta I, Salgado D. 2015. Determination of piglets' rectal temperature and respira- tory rate through skin surface temperature under climate chamber conditions. Eng Agric 3: 979-989. doi: 10.1590/18094430-Eng.Agric.v35n6p979-989/2015

14. [OIE] Organización Mundial de Sanidad Animal. s.f. C[odigos y manuals. [Internet]. Disponible en: https:// www.oie.int/es/que-hacemos/normas/ codigos-y-manuales/

15. Poikalainen V, Praks J, Veermäe I, Kokin E. 2012. Infrared temperature patterns of cow's body as an indicator for health control at precision cattle farming. Agron Res Biosys Engin 1: 187-194.

16. Sellier N, Guettier E, Staub C. 2014. A review of methods to measure animal body temperature in precision farming. Am J Agric Sci Technol 2: 74-99.

17. Siguayro R. 2009. Comparación de las características físicas de las fibras de la llama Ch'aku (Lama glama) y la alpaca huacaya (Lama pacos) del centro experimental Quimsachata del INIA Puno. Tesis de Maestría. Lima, Perú: Univ. Nacional Agraria La Molina. 63 p.

18. Soerensen D, Pedersen L. 2015. Infrared skin temperature measurements for monitoring health in pigs: a review. Acta Vet Scand 57: 5. doi: 10.1186/ s13028-015-0094-2

19. Tabuaciri P, Bunter K, Graser H. 2012. Thermal imaging as a potential tool for identifying piglets at risk. In: AGBU Pig Genetics Workshop. Australia.

20. Vieira M, Correa S, Salles F, Roma L, El Faro L, Bustos P, et al. 2016. Mapping the body surface temperature of cattle by infrared thermography. $\mathrm{J}$ Therm Biol 62: 63-69. doi: 10.1016/ j.jtherbio.2016.10.003

21. Vilela J. 2018. Estudio comparativo preliminar de la medición de temperatura corporal vía rectal y vía infrarroja en alpacas (Vicugna pacos). En: VIII Congreso Mundial de Camélidos. Oruro, Bolivia. 